\title{
Isolated Right Ventricle Mass: The Role of Echocardiography in Diagnostic Dilemma
}

\author{
Suruchi Ladha ${ }^{1} \quad$ Usha Kiran ${ }^{1}$ Santanu Kumar Bora ${ }^{1}$ \\ ${ }^{1}$ Department of Cardiac Anaesthesia, Cardiothoracic Centre, \\ All India Institute of Medical Sciences, New Delhi, India
}

\begin{abstract}
Address for correspondence Suruchi Ladha, Senior Resident, Department of Cardiac Anaesthesia, 7th Floor, Cardiothoracic Centre, All India Institute of Medical Sciences, Ansari Nagar, New Delhi 110029, India (e-mail: suruchi.ladha@gmail.com).
\end{abstract}

\begin{abstract}
Keywords

- right ventricle

- echocardiography

- mass

- diagnosis

The presence of a right ventricle mass is uncommon, especially if there is no associated mass in the other chambers of the heart. Right ventricle mass is usually due to thrombi or vegetations; however, a variety of tumors may also present similarly. The authors report the presence of an isolated right ventricle mass in a young female patient and the intraoperative use of transesophageal echocardiography in diagnostic dilemma. Echocardiography helps in assessing the characteristics of the mass, such as the size, shape, attachment points, and composition, and continues to remain an invaluable tool for guiding both the anesthetic and the surgical management of the case.
\end{abstract}

\section{Introduction}

Echocardiography is a vital imaging technique for the assessment of intracardiac mass. ${ }^{1,2}$ The presence of a right ventricle (RV) mass, especially when not associated with any mass in the right atrium, pulmonary arteries, or left ventricle, is a rare clinical scenario. ${ }^{3}$ The role of echocardiography is critical in patients having isolated RV mass to guide the treatment decisions for prognostication. The common differential diagnosis of isolated RV cardiac mass can be a tumor, thrombus, or vegetation..$^{1,2}$

The characterization of RV mass by echocardiography is not straightforward. Echocardiography can be used to narrow the differential diagnosis of cardiac masses by delineation of important mass characteristics such as the size, shape, attachment points, homogeneity, presence of necrotic areas, and echolucencies. ${ }^{1}$ We report a case of isolated RV mass scheduled for surgical excision and thereby discuss the role of the transesophageal echocardiography in the diagnosis and management of the case.

\section{Case Report}

A 20-year-old female presented with 2-week history of shortness of breath, chest pain, and hemoptysis. There was past history of hospitalization; 1 year previously for headache and blurring of vision during which magnetic resonance imaging of the brain was suggestive of cerebral venous thrombosis. However, the etiology of thrombosis could not be established. The patient had taken an opinion from the Department of Neurosurgery and Hematology. She was receiving oral medications: warfarin (7.5 $\mathrm{mg}$ once daily), levetiracetam (250 mg twice daily), dexamethasone (4 mg thrice daily), and clonazepam ( $0.25 \mathrm{mg}$ once daily) as per the neurosurgical opinion. On examination, the patient had a pulse rate of $88 / \mathrm{min}$; blood pressure of $114 / 70 \mathrm{~mm} \mathrm{Hg}$; cardiovascular system- S1 and S2 well heard, and systolic murmur was heard in the subxiphoid area. On investigation, the coagulation profile was deranged and hence warfarin was withheld. Transthoracic echocardiography showed large mass in RV $(3.7 \times 1.3 \mathrm{~cm})$, severe tricuspid regurgitation (TR), moderate pulmonary artery hypertension (PAH), and no mass in right atrium or pulmonary arteries with normal biventricular function. Computed tomography (CT) scan of chest showed enlarged, necrotic, bilateral hilar, and preand paratracheal lymph nodes with hypo dense focus in RV. Whole body positron emission tomography (PET)-CT showed metabolically active, necrotic, bilateral hilar, and mediastinal lymph nodes most likely infective in origin. The mass in the $\mathrm{RV}$, however, did not show increased uptake. Fine-needle aspiration cytology from the mediastinal lymph nodes was attempted but was inconclusive. Lower limb Doppler was performed, but there was no evidence of any thrombus in the lower limb circulation. Patient was started on enoxaparin 
but there was no symptomatic improvement. Subsequently, surgical management was planned.

Intraoperative transesophageal echocardiography showed relatively immobile RV mass of size around $4 \times 2 \times 1.7 \mathrm{~cm}$ toward apex (-Figs. 1-3). It was found to have attachment to the anterior leaflet of the tricuspid valve ( - Fig. 4). Patient had severe TR with eccentric jet (-Fig. 5).

Patient had moderate $\mathrm{PAH}$ with right ventricular systolic pressure of $48 \mathrm{~mm} \mathrm{Hg}$. There was nonsignificant RV outflow tract gradient. TAPSE measurement showed a value of $16 \mathrm{~mm}$. The RV end-diastolic measurement was $\sim 38 \mathrm{~mm}$ at the base and the longitudinal dimension was $74 \mathrm{~mm}$. Incidental patent foramen ovale (PFO) was detected ( - Fig. 6).

The echocardiographic examination of the RV mass revealed that it was a solitary mass, isolated in the RV; there was no associated mass in the right atrium, pulmonary artery, or left ventricle; it was a relatively immobile mass, homogenous in appearance, with a broad-based attachment to the RV free wall and apex; it was also attached to the tricuspid valve anterior leaflet; and there were no areas of necrosis or echolucencies in the mass. These favored a echocardiography-based diagnosis of an isolated RV thrombus with no embolization to the pulmonary arteries. During surgery, the thrombus was excised and as the tricuspid leaflet was found adherent to it,

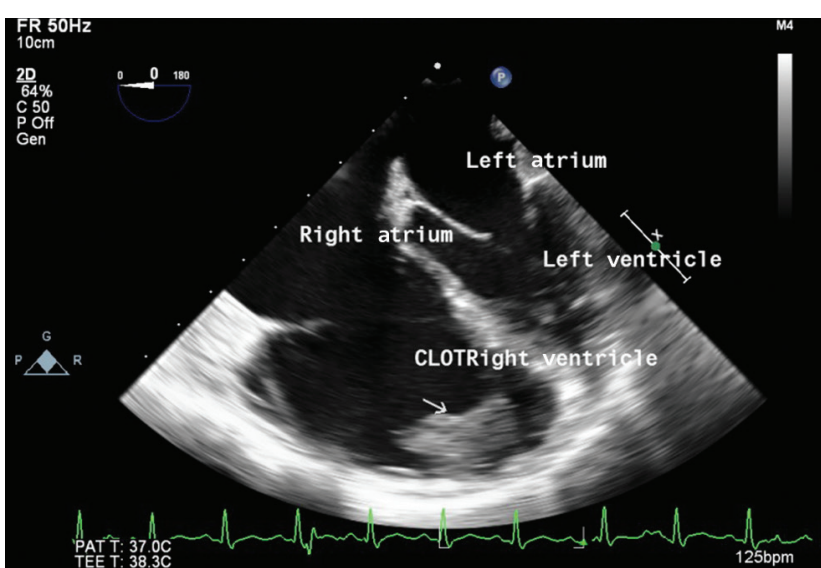

Fig. 1 Mid-esophageal four-chamber view showing thrombus in right ventricle.

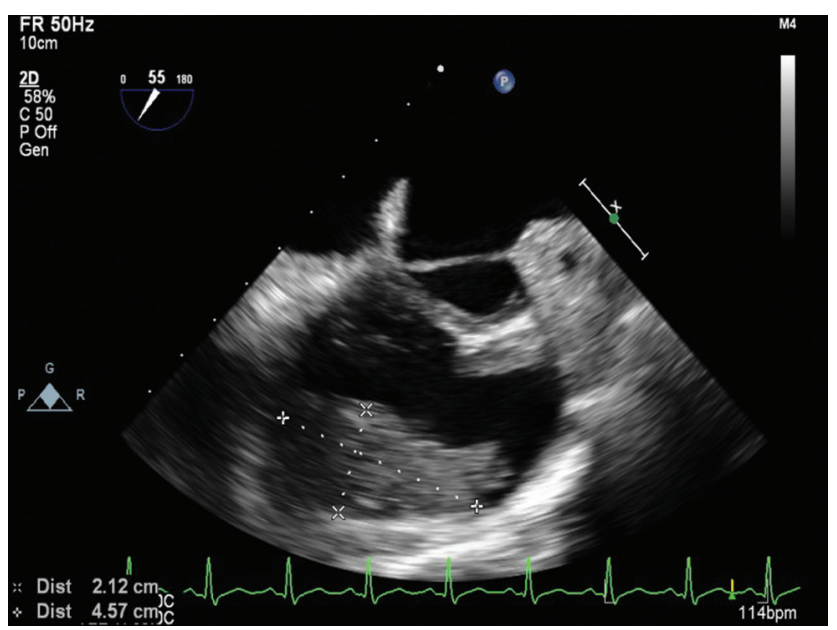

Fig. 2 Mid-esophageal aortic valve short-axis view. tricuspid valve repair was done. After the surgery, patient was weaned off bypass smoothly. Postoperatively, the echocardiographic examination revealed the complete removal of the thrombus and there was no significant TR. The resected mass was sent for histopathological examination. Microscopic examination revealed partially organized thrombus and there was no diagnostic feature of cardiac myxoma. The patient had an uneventful postoperative recovery.

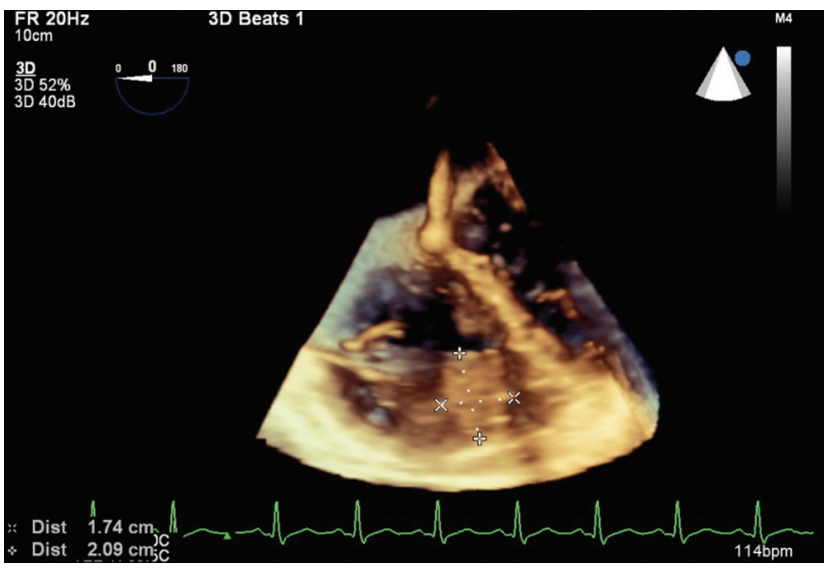

Fig. 3 Three-dimensional reconstruction showing mass measurement.

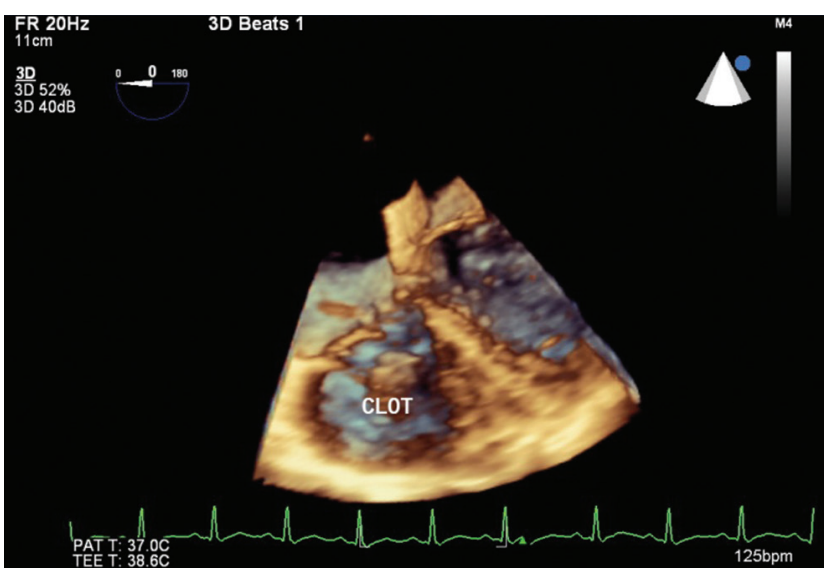

Fig. 4 Three-dimensional reconstruction showing tricuspid valve attachment (anterior leaflet of tricuspid valve).

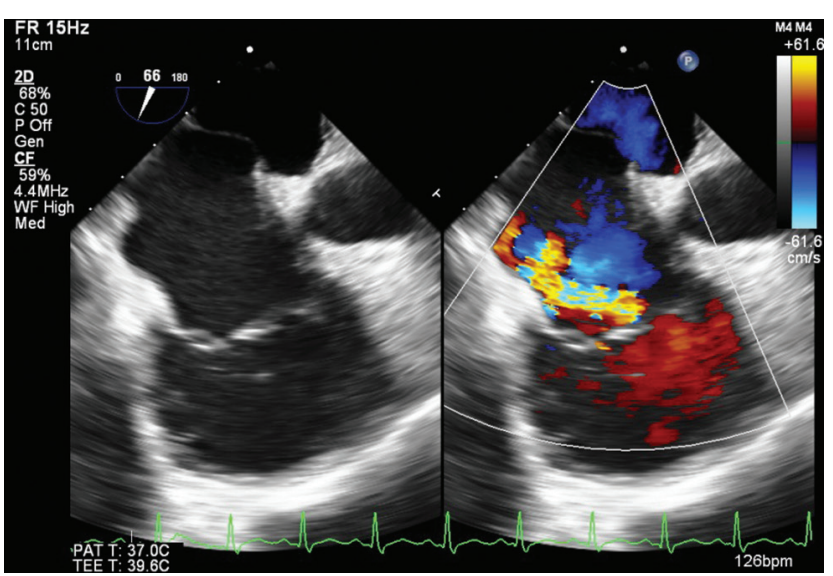

Fig. 5 Mid-esophageal right ventricular inflow-outflow view showing eccentric jet across tricuspid valve. 


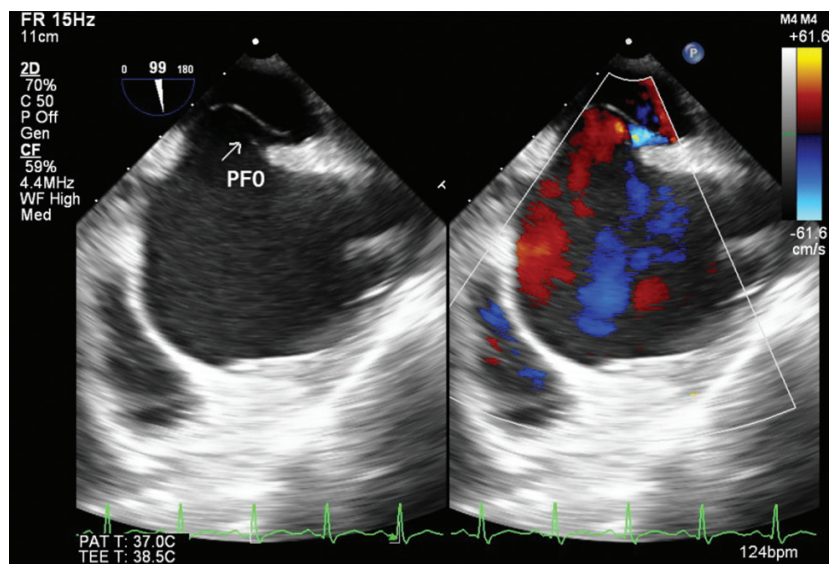

Fig. 6 Modified bicaval view showing patent foramen ovale (PFO).

\section{Discussion}

A detailed echocardiographic examination can assist in situations of diagnostic dilemma. The main challenge in our case was differentiating cardiac thrombus from tumors, especially myxoma. The presence of either a RV thrombus or tumor, especially in isolation, is a rare situation.

Right ventricle thrombus has a relatively rare incidence. ${ }^{4}$ Three patterns of right heart thrombi have been identified by the European Working Group on Echocardiography. ${ }^{5}$ Type A thrombi are believed to be emboli from large veins. Predisposing factors for these thromboemboli include prominent Eustachian valves, TR, low cardiac output, and pulmonary hypertension. ${ }^{6}$ They are serpiginous masses. They can prolapse into tricuspid or pulmonary valve during cardiac cycle. They are usually mobile structures freely floating in the chamber with minimal or nil point of attachment. Type B thrombi are mural thrombi which are formed in situ. They are found in low cardiac output condition in association with underlying cardiac abnormalities. These are mural thrombi with reduced motion during the cardiac cycle, a broad-based attachment to the heart wall, and occasional focal calcification. ${ }^{5}$ Type $C$ thrombi are uncommon and are highly mobile.

Metastatic tumors that invade the heart occur 20 to 40 times more frequently than primary tumors. Primary cardiac tumors are uncommon with incidence of $0.02 \%$. Of the primary cardiac tumors, $\sim 75 \%$ are benign, and of these benign tumors, myxomas are the most common. ${ }^{7}$ The chamber predilection of myxoma is left atrium (75\%), followed, in order of prevalence, by the right atrium, left ventricle, and the last is RV. The remaining primary cardiac tumors are malignant, the majority of which include sarcomas. Cardiac sarcomas arise from the right side of the heart in around 25\% of reported cases. ${ }^{7}$ RV myxomas typically present with triad of embolism, intracardiac obstruction, and systemic symptoms such as syncope and exertional dyspnea.

Our patient had a history of cerebral thrombosis, and the intraoperative echocardiographic finding suggested a relatively immobile mass with a broad-based attachment favoring diagnosis of thrombus. In our case, real-time threedimensional echocardiography further helped in the assessment of thrombus mobility, differentiation between thrombus and myocardium, and delineation of changes in thrombi structure and assessment of the thrombus volume. On echocardiographic examination, myxoma usually has a narrow base of attachment to the cardiac free wall, is mobile, and it can be heterogeneous in appearance with areas of hemorrhage, necrosis, cyst formation, and calcification. ${ }^{8}$ The presence of irregular texture to the mass also suggests that it is a tumor as opposed to a thrombus. The absence of these features in our case helped us in differentiating it from myxoma. Furthermore, there was no family history, or any constitutional symptoms. Many authors have reported that RV myxoma usually presents with RV outflow obstruction, which was not seen in our patient. ${ }^{9}$ Vegetations in the RV can appear as single or multiple, linear, round, or as a mass with nonuniform border with high frequency flutter or oscillations, which is mostly attached to valves. Absence of fever, constitutional symptoms, and echocardiographic findings like oscillations rules out vegetations.

Echocardiographic examination also helped in the diagnosis of an incidental PFO. In such patients, a PFO can lead to systemic embolization. Right heart thrombus is frequently associated with major pulmonary thromboembolism, but is rarely recognized before a catastrophic event like death. In our case, the echocardiographic examination ruled out the presence of any thrombus which might have embolized to the pulmonary artery, so the pulmonary artery was not opened surgically. The choice of an optimal surgical access prior to removal of intracardiac mass can also be guided by echocardiography. During surgery, the anterior leaflet of tricuspid valve was found to be attached to the thrombus and repair of the valve was done. On tissue histopathology, it was found to be a partly organized thrombus.

The presence of RV thrombus has been described in the setting of autoimmune diseases, hypercoagulability states, cardiomyopathy, right ventricular pacing, and right ventricular infarction. In our case, the etiology of the RV thrombus could not be determined. In the Indian subcontinent, tuberculosis is considered to be an important risk factor for repeated thromboembolic events because of the high prevalence of the disease and also it can have varied clinical presentations. ${ }^{10}$ Although tuberculosis was not confirmed in our case as the histopathology was negative, the RV thrombus cannot be attributed to tuberculosis. However, the hilar lymphadenopathy, hemoptysis, and fibrotic changes in lungs on CT scan with repeated thrombus formation lead to a suspicion of possible tuberculosis and the patient was started on antituberculosis treatment in the postoperative period. The incidence of cardiac and systemic thromboembolism in tuberculosis is assumed to be rare, and in a few case series the incidence of deep venous thrombosis has been associated with 1.5 to $3.4 \%$ cases of tuberculosis. ${ }^{10}$

\section{Conclusion}

Isolated RV cardiac mass is a rare clinical condition, which leads to a diagnostic dilemma, and echocardiography remains an invaluable tool in perioperative diagnosis and management. Transesophageal echocardiography provides excellent 
cardiac imaging and helps in both the anesthesia and surgical management of the case.

\section{Conflict of Interest}

None.

\section{Financial Disclosures}

None.

\section{References}

1 Zaragoza-Macias E, Chen MA, Gill EA. Real time threedimensional echocardiography evaluation of intracardiac masses. Echocardiography 2012;29(2):207-219

2 Müller S, Feuchtner G, Bonatti J, et al. Value of transesophageal 3D echocardiography as an adjunct to conventional 2D imaging in preoperative evaluation of cardiac masses. Echocardiography 2008;25(6):624-631

3 Wertman BM, Goland S, Davidson RM, Siegel RJ. Right atrial and ventricular masses of unknown origin. J Am Soc Echocardiogr 2008;21(6):776.e5-776.e7

4 Waller BF, Grider L, Rohr TM, McLaughlin T, Taliercio CP, Fetters J. Intracardiac thrombi: frequency, location, etiology, and complications: a morphologic review-Part IV. Clin Cardiol 1995;18(11):669-674

5 European Working Group on Echocardiography. The European Cooperative Study on the clinical significance of right heart thrombi. Eur Heart J 1989;10(12):1046-1059

6 Mohan B, Chhabra ST, Gulati A, et al. Clinical and echocardiographic diagnosis, follow up and management of right-sided cardiac thrombi. Indian Heart J 2013;65(5):529-535

7 Gopal AS, Arora NS, Messineo FC. Right ventricular myxoma. N Engl J Med 2000;342(4):295

8 Lebovic S, Koorn R, Reich DL. Role of two-dimensional transoesophageal echocardiography in the management of a right ventricular tumour. Can J Anaesth 1991;38(8):1050-1054

9 Chen C, Gu J, Meng W, et al. Intraoperative evaluation of right ventricularoutflowtractmyxomabyrealtimethree-dimensional transesophageal echocardiography. Echocardiography 2014; 31(6):E174-E176

10 Gupta A, Mrigpuri P, Faye A, Bandyopadhyay D, Singla R. Pulmonary tuberculosis - an emerging risk factor for venous thromboembolism: a case series and review of literature. Lung India 2017;34(1):65-69 